\title{
Oxidative stress biomarkers in Fabry disease: is there a room for them?
}

\author{
C. Simoncini ${ }^{1}$ - S. Torri ${ }^{1}$. V. Montano ${ }^{1} \cdot$ L. Chico $^{1} \cdot$ F. Gruosso ${ }^{1} \cdot$ A. Tuttolomondo ${ }^{2} \cdot$ A. Pinto $^{2} \cdot$ I. Simonetta $^{2}$.

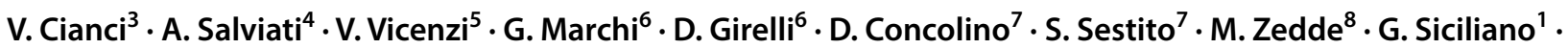 \\ Michelangelo Mancuso ${ }^{1}$
}

Received: 22 April 2020 / Revised: 14 June 2020 / Accepted: 1 July 2020 / Published online: 27 July 2020

(c) The Author(s) 2020

\begin{abstract}
Background Fabry disease (FD) is an X-linked lysosomal storage disorder, caused by deficient activity of the alpha-galactosidase A enzyme leading to progressive and multisystemic accumulation of globotriaosylceramide. Recent data point toward oxidative stress signalling which could play an important role in both pathophysiology and disease progression.

Methods We have examined oxidative stress biomarkers [Advanced Oxidation Protein Products (AOPP), Ferric Reducing Antioxidant Power (FRAP), thiolic groups] in blood samples from 60 patients and 77 healthy controls.

Results AOPP levels were higher in patients than in controls $(p<0.00001)$ and patients presented decreased levels of antioxidant defences (FRAP and thiols) with respect to controls $(p<0.00001)$. In a small group of eight treatment-naïve subjects with FD-related mutations, we found altered levels of oxidative stress parameters and incipient signs of organ damage despite normal lyso-Gb3 levels.

Conclusions Oxidative stress occurs in FD in both treated and naïve patients, highlighting the need of further research in oxidative stress-targeted therapies. Furthermore, we found that oxidative stress biomarkers may represent early markers of disease in treatment-naïve patients with a potential role in helping interpretation of FD-related mutations and time to treatment decision.
\end{abstract}

Keywords Fabry disease $\cdot$ Biomarkers $\cdot$ lysoGb3 $\cdot$ Oxidative stress

Michelangelo Mancuso

michelangelo.mancuso@unipi.it

1 Department of Clinical and Experimental Medicine, Neurological Institute, University of Pisa, Pisa, Italy

2 Internal Medicine and Stroke Care Ward, Department of Promoting Health, Maternal-Infant, Excellence and Internal and Specialized Medicine (Promise) G. D'Alessandro, University of Palermo, Palermo, Italy

3 Regional Epilepsy Centre, Great Metropolitan Hospital "Bian chi-Melacrino-Morelli”, Reggio Calabria, Italy

4 Lab Functional Genomics, Department of Biotechnology, Univ Verona, Genartis srl, Verona, Italy

5 Medical Genetics Unit, ASL 9, Verona, Italy

6 Internal Medicine Unit, Azienda Ospedaliera Universitaria Integrata di Verona, Verona, Italy

7 Department of Medical and Surgical Sciences, Pediatric Unit, "Magna Graecia" University, Catanzaro, Italy

8 Azienda Unità Sanitaria Locale, IRCCS di Reggio Emilia, Reggio Emilia, Italy

\section{Introduction}

Fabry disease (FD) is an X-linked lysosomal storage disorder, caused by deficient activity of the alpha-galactosidase A enzyme (GAL-A), encoded by the GLA gene. The enzyme deficiency causes accumulation of the sphingolipid globotriaosylceramide (GL-3 or Gb3) and its deacylated derivative lyso-globotriaosylceramide (lyso-GL-3 or lyso-Gb3) in various cell types, in particular the vascular endothelial and the smooth muscle cells, cardiac myocytes, dorsal root ganglion neurons, neurons of the autonomic nervous system, brain and all types of kidney cells [1].

Although male patients are traditionally considered to develop a more severe phenotype than females, the disorder has a substantial clinical heterogeneity. The classical phenotype (type 1) exhibits early manifestations, whereas non-classical or late-onset phenotype (type 2) presents with clinical signs later in life [1]. In a multicentre study [2], classical FD was differentiated from non-classical FD by the presence of at least one of three clinical criteria 
(neuropathic pain, angiokeratoma, and cornea verticillata) and an additional biochemical criterion in males (leukocyte GAL-A activity $<5 \%$ ). Although several mutations in $G L A$ have been associated to classic or late-onset forms, a definite genotype-phenotype correlation is not demonstrated, and diagnosis and classification should not be made only on genetic-basis.

Substrates deposition is related to tissue damage in FD; however, the underlying molecular mechanisms remain not completely understood. Recent data highlighted the role of oxidative stress in FD pathophysiology. FD patients present high lipid and protein oxidative damage, decreased antioxidant defences and increased inflammatory biomarkers and cytokines [3-10]. Excess intracellular Gb3 induces oxidative stress and up-regulates the expression of cellular adhesion molecules in vascular endothelial cells [5]. Moreover, it has been suggested that pro-oxidant state occurs, is correlated and seems to be induced by Gb3 in Fabry patients [9].

\section{Hypothesis/study objective/purpose}

The aim of our multicentre project was to evaluate the role of oxidative stress in FD. In particular, we have evaluated (i) if oxidative stress occurs in blood; (ii) if there is an association between oxidative stress biomarkers and FD clinical manifestations and lor a difference between classic and late onset FD; (iii) if oxidative stress parameters over time are related with Lyso-Gb3 and disease appearance or progression, in a subgroup of eight treatment-naïve subjects/patients with normal Lyso-Gb3 levels, in order to see if selected oxidative stress biomarkers could represent early markers of disease progression.

\section{Materials and methods}

The patients group consisted of 60 Caucasian genetically proven FD subjects (Table 1) from Italy recruited from seven Italian centres with expertise in FD. Diagnosis and phenotype classification was performed according to international recommendations [1,2], although few cases remained of uncertain interpretation as discussed below.

All the subjects had $10 \mathrm{ml}$ of blood drawn from an antecubital vein in the morning (at fast) for analysis of the advanced oxidation protein products (AOPP), antioxidant ferric-reducing power (FRAP), and total thiol groups.

The results were compared to those obtained from a population of 77 healthy controls ( 32 men, mean age $61.5 \pm 18.2$ years), unrelated to the patients, who represent the normative values at the coordinator centre.

AOPP, a marker of oxidative damage to proteins, were assessed according to Witko-Sarsat et al. [11].
AOPP are well-known biomarkers used to evaluate the oxidative modification of proteins, which occurs through myeloperoxidase (MPO) activity of neutrophils. MPO catalyses the production of hypochlorous acid from hydrogen peroxide and chloride ions; hypochlorous acid is capable of oxidizing plasma proteins to generate AOPP. AOPP levels correlated with plasma concentrations of dityrosine and advanced glycation end-products (AGE)-pentosidine as indices of oxygen-mediated protein damage. AOPP measure highly oxidized proteins, especially albumin, and reflect the protein oxidation derived from neutrophils and monocytes [12]. Moreover, it appears that AOPP are integral part of the non-enzymatic antioxidant system of plasma proteome. Oxidized fibrinogen, a molecule responsible for the positivity of AOPP chemical reaction, is bound to apolipoprotein(a), which could compete with plasminogen for its binding sites on fibrinogen, causing inhibition of fibrinolysis and promoting cardiovascular damage. Though it is suggested that the AOPP system functions as a double-edged weapon of the plasma proteome, it by no means diminishes its diagnostic value as an oxidative stress biomarker [13]. For this reason and considering that oxidative modification products of proteins have several advantages such as early formation, longer lifespan, greater stability and reliability in comparison with other oxidative markers [13], we have chosen AOPP as biomarker to test if protein-oxidative changes occur in FD.

In order to measure non-enzymatic anti-oxidant properties, FRAP was assessed in plasma samples, according to Benzie and Strain [14]. FRAP reflects the concentration of low molecular-weight non-enzymatic antioxidants and is independent of the concentration of proteins [15]. We selected FRAP assay for this study because it gives fast and reproducible results, permitting, at the same time, a more objective evaluation of the non-protein antioxidant activity.

The content of plasmatic total thiols (t-SH) was estimated by evaluation of the sulfhydryl groups present in the molecules, following the protocol described by $\mathrm{Hu}$ [16]. Sulfhydryl groups (-SH) are responsible for maintaining the structure and function of proteins, enzymes and membranes as well as they can decrease the damage caused by oxidative stress.

Finally, lyso-Gb3 was analysed on dried blood spot (DBS) on different laboratories and data acquired at the coordinator centre for analysis.

We did not perform any statistical analysis between AGAL-A activity in peripheral blood leukocytes and oxidative stress parameters because in our cohort most of the cases are female (66\%), and in female AGAL-A activity is not a reliable marker of disease [1].

In a subgroup of eight patients in whom the diagnosis of FD was made being relatives of symptomatic cases, we have monitored oxidative stress biomarkers, Lyso-Gb3 and eventual pre-clinical organ involvement over time. 
Table 1 demographic, molecular and clinical features of the FD patients enrolled in the study

\begin{tabular}{|c|c|c|c|c|c|}
\hline & Gender & Mutation & $\begin{array}{l}\text { Age of } \\
\text { onset } \\
\text { (years) }\end{array}$ & Clinical onset & Clinical involvement at last follow-up \\
\hline 1 & $\mathrm{~F}$ & c. $337 T>$ C p.Phe113Leu & 33 & - & - \\
\hline 2 & $\mathrm{~F}$ & c.337T > C p.Phe113Leu & 27 & - & - \\
\hline 3 & M & c.337T > C p.Phe113Leu & 62 & Myocardial infarction & HCM, Arrhythmia, Myocardial infarction \\
\hline 4 & M & deletion exons 3,4 & 9 & Acroparesthesia & Acroparesthesia, HCM \\
\hline 5 & M & c.337T > C p.Phe113Leu & 62 & Arrhythmia & Arrhythmia, HCM, Proteinuria, VBD \\
\hline 6 & $\mathrm{~F}$ & deletion exons 3,4 & 50 & Acroparesthesia & Acroparesthesia \\
\hline 7 & M & c. $818 \mathrm{~T}>$ C p.Phe273Ser & 22 & Acroparesthesia & $\begin{array}{l}\text { Acroparesthesia, HCM, Arrhythmia, Pro- } \\
\text { teinuria, Stroke, VBD }\end{array}$ \\
\hline 8 & $\mathrm{~F}$ & c.337 T > C p.Phe113Leu & 40 & Stroke & Stroke, Proteinuria \\
\hline 9 & $\mathrm{~F}$ & c. $.550 \mathrm{~T}>\mathrm{A}$ & 39 & $\mathrm{HCM}$ & HCM, Stroke \\
\hline 10 & $\mathrm{~F}$ & $\begin{array}{l}\text { IVS2-76_80 del15; IVS 4-16 A > G; IVS 6-22 } \\
\text { G > T }\end{array}$ & 30 & Acroparesthesia & Acroparesthesia, Stroke \\
\hline 11 & $\mathrm{~F}$ & $\begin{array}{l}.-10 \mathrm{G}>\mathrm{T} \text {, IVS 6-22 C > T; IVS 6-51_54 } \\
\text { del14, g.9151 C > T }\end{array}$ & 32 & Acroparesthesia & $\begin{array}{l}\text { Acroparesthesia, Proteinuria, Stroke, GI } \\
\text { disorders }\end{array}$ \\
\hline 12 & M & $\begin{array}{l}.-10 \mathrm{C}>\mathrm{T} \text {; IVS 2-76_80 del 5; IVS 4-16 } \\
\text { A > G; IVS 6-22 C > T }\end{array}$ & 4 & GI disorders & GI disorders, Acroparesthesia \\
\hline 13 & $\mathrm{~F}$ & $.-30 \mathrm{G}>\mathrm{A}$ & 30 & Acroparesthesia & Acroparesthesia, Stroke, GI disorders \\
\hline 14 & $\mathrm{~F}$ & $\begin{array}{l}.-10 \mathrm{C}>\mathrm{T} \text {; IVS 2-76_80 del 5; IVS 4-16 } \\
\quad \mathrm{A}>\mathrm{G} \text {; IVS 6-22 C > T }\end{array}$ & 35 & Stroke & Stroke, Acroparesthesia \\
\hline 15 & $\mathrm{~F}$ & c.547 G>A p.G183S & 17 & Acroparesthesia & Acroparesthesia, Proteinuria \\
\hline 16 & M & c.614delC & 29 & $\mathrm{HCM}$ & HCM, Acroparesthesia \\
\hline 17 & $\mathrm{~F}$ & c.614delC & 12 & Acroparesthesia & Acroparesthesia, Stroke, HCM \\
\hline 18 & $\mathrm{~F}$ & $\begin{array}{l}.-10 \mathrm{C}>\mathrm{T} ; \text { IVS 2-76_80 del 5; IVS 4-16 } \\
\text { A > G; IVS 6-22 C > T }\end{array}$ & 30 & Acroparesthesia & Acroparesthesia \\
\hline 19 & M & $\begin{array}{l}.-10 \text { C > T; IVS 2-76_80 del 5; IVS 4-16 } \\
\quad \text { A > G; IVS 6-22 C > T }\end{array}$ & 10 & GI disorders & GI disorders, Acroparesthesia, Proteinuria \\
\hline 20 & $\mathrm{~F}$ & c. $547 \mathrm{G}>$ A p.G183S & 22 & - & - \\
\hline 21 & M & c.337 T > C p.Phe113Leu & 40 & Proteinuria & Proteinuria, HCM \\
\hline 22 & $\mathrm{~F}$ & c. $1146 \mathrm{C}>\mathrm{A} ;$ p.C $382 \mathrm{X}$ & 12 & Proteinuria & Proteinuria \\
\hline 23 & $\mathrm{~F}$ & p.Tyr222Asp & 49 & Proteinuria & Proteinuria, Arrhythmia, GI disorders \\
\hline 24 & M & c. $640-859 \mathrm{C}>\mathrm{T}$ & 32 & $\mathrm{HCM}$ & $\begin{array}{l}\text { HCM, Arrhythmia, Proteinuria, VBD, GI } \\
\text { disorders }\end{array}$ \\
\hline 25 & $\mathrm{~F}$ & D313Y & 31 & VBD & VBD, GI disorders \\
\hline 26 & $\mathrm{~F}$ & p.Ala377Asp & 32 & Acroparesthesia & Acroparesthesia, Arrhythmia, Proteinuria \\
\hline 27 & $\mathrm{~F}$ & c. $1146 \mathrm{C}>\mathrm{A} ;$ p.C $382 \mathrm{X}$ & 30 & Acroparesthesia & Acroparesthesia, TIA, VBD, GI disorders \\
\hline 28 & $\mathrm{~F}$ & c. $640-859 \mathrm{C}>\mathrm{T}$ & 52 & Proteinuria & Proteinuria, HCM, Arrhythmia, VBD \\
\hline 29 & $\mathrm{~F}$ & p.Tyr222Asp & 13 & Acroparesthesia & Acroparesthesia, VBD, GI disorders \\
\hline 30 & $\mathrm{~F}$ & D313Y & 36 & cSVD & cSVD, VBD, Proteinuria \\
\hline 31 & $\mathrm{~F}$ & $\begin{array}{l}\text { p.Ala143Thr } \\
\text { p.Leu394Pro }\end{array}$ & 41 & - & - \\
\hline 32 & $\mathrm{~F}$ & $\begin{array}{l}\text { p.Ala143Thr } \\
\text { p.Leu394Pro }\end{array}$ & 41 & Stroke & Stroke \\
\hline 33 & M & c. $818 \mathrm{~T}>$ C p.Phe273Ser & 62 & $\mathrm{HCM}$ & $\begin{array}{l}\text { HCM, Arrhythmia, Proteinuria, cSVD, GI } \\
\text { disorders }\end{array}$ \\
\hline 34 & M & $\mathrm{N} 215 \mathrm{~S}$ & 64 & $\mathrm{HCM}$ & HCM, Arrhythmia \\
\hline 35 & $\mathrm{~F}$ & c. $334 \mathrm{C}>\mathrm{T}$ p.Arg 112 Cys & 62 & Proteinuria & Proteinuria, HCM, Arrhythmia, cSVD, VBD \\
\hline 36 & $\mathrm{~F}$ & c. $334 \mathrm{C}>\mathrm{T}$ p.Arg $112 \mathrm{Cys}$ & 70 & Arrhythmia & Arrhythmia, HCM, cSVD, VBD \\
\hline 37 & M & c. $334 \mathrm{C}>\mathrm{T}$ p.Arg $112 \mathrm{Cys}$ & 37 & Proteinuria & $\begin{array}{l}\text { Proteinuria, HCM, cSVD, VBD, Acropares- } \\
\text { thesia }\end{array}$ \\
\hline 38 & $\mathrm{~F}$ & c. $334 \mathrm{C}>\mathrm{T}$ p.Arg $112 \mathrm{Cys}$ & 33 & Acroparesthesia & Acroparesthesia \\
\hline
\end{tabular}


Table 1 (continued)

\begin{tabular}{|c|c|c|c|c|c|}
\hline & Gender & Mutation & $\begin{array}{l}\text { Age of } \\
\text { onset } \\
\text { (years) }\end{array}$ & Clinical onset & Clinical involvement at last follow-up \\
\hline 39 & M & p.Cys63Tyr & 46 & Acroparesthesia & $\begin{array}{l}\text { Acroparesthesia, HCM, Arrhythmia, CKD, } \\
\text { Stroke }\end{array}$ \\
\hline 40 & $\mathrm{~F}$ & p.Arg118Cys & 49 & - & - \\
\hline 41 & M & p.Arg118Cys & 18 & $\mathrm{HCM}$ & $\mathrm{HCM}$ \\
\hline 42 & $\mathrm{~F}$ & c.97G > T p.Asp33Tyr & 51 & GI disorders & GI disorders, HCM \\
\hline 43 & M & c. $851 \mathrm{~T}>\mathrm{C}$ p.Met $284 \mathrm{Thr}$ & 34 & Proteinuria & Proteinuria, HCM, Arrhythmia, CKD \\
\hline 44 & $\mathrm{~F}$ & c.113delG & 6 & Acroparesthesia & Acroparesthesia, Proteinuria, TIA \\
\hline 45 & $\mathrm{~F}$ & c. $113 \mathrm{delG}$ & 8 & GI disorders & GI disorders, Proteinuria, Acroparesthesia \\
\hline 46 & $\mathrm{~F}$ & c. $335 \mathrm{G}>\mathrm{A} \operatorname{Arg} 112 \mathrm{His}$ & 22 & Proteinuria & Proteinuria \\
\hline 47 & $\mathrm{~F}$ & c. $860 \mathrm{G}>\mathrm{A}$ & 8 & Acroparesthesia & $\begin{array}{l}\text { Acroparesthesia, HCM, Proteinuria, GI } \\
\text { disorders }\end{array}$ \\
\hline 48 & $\mathrm{~F}$ & c. $860 \mathrm{G}>\mathrm{A}$ & 49 & GI disorders & GI disorders, HCM, Proteinuria \\
\hline 49 & $\mathrm{~F}$ & c. $779 \mathrm{G}>\mathrm{A}$ & 56 & Proteinuria & Proteinuria, HCM \\
\hline 50 & $\mathrm{~F}$ & c. $846 \_847 \mathrm{delTC}$ & 18 & Proteinuria & Proteinuria \\
\hline 51 & M & c. $846 \_847 \mathrm{delTC}$ & 17 & Proteinuria & Proteinuria, $\mathrm{HCM}$ \\
\hline 52 & $\mathrm{~F}$ & c. $846 \_847 \mathrm{delTC}$ & 15 & Proteinuria & Proteinuria, PM, TIA \\
\hline 53 & M & $\mathrm{C} 172 \mathrm{Y}$ & 12 & GI disorders & GI disorders, Proteinuria \\
\hline 54 & $\mathrm{~F}$ & $\mathrm{C} 172 \mathrm{Y}$ & 20 & Proteinuria & Proteinuria, HCM \\
\hline 55 & M & $\mathrm{C} 172 \mathrm{Y}$ & 23 & Proteinuria & Proteinuria \\
\hline 56 & M & c. $846 \_847 \mathrm{delTC}$ & 62 & Proteinuria & Proteinuria \\
\hline 57 & M & $\mathrm{C} 172 \mathrm{Y}$ & 14 & GI disorders & GI disorders, HCM, Proteinuria \\
\hline 58 & $\mathrm{~F}$ & c. $846 \_847 \mathrm{delTC}$ & 25 & Stroke & Stroke, HCM, PM, Proteinuria, TIA \\
\hline 59 & $\mathrm{~F}$ & c. $846 \_847 \mathrm{delTC}$ & 12 & Acroparesthesia & Acroparesthesia, Proteinuria \\
\hline 60 & $\mathrm{~F}$ & $\mathrm{C} 172 \mathrm{Y}$ & 5 & - & - \\
\hline
\end{tabular}

$H C M$ hypertrophic cardiomyopathy, $c S V D$ cerebral small vessel disease, $V B D$ vertebrobasilar dolichoectasia, $C K D$ chronic kidney disease, $P M$ PaceMaker, GI gastrointestinal

During this observational time, no additional therapies were undertaken, lifestyle did not change, and no unrelated FD diseases occurred.

The Ethic Committees of each centre approved the study, and patients provided informed consent to participate in this study.

\section{Statistical analysis}

Statistical analysis was performed using SPSS Statistics (Statistical Package for Social Science, 20.0 version for Windows). Quantitative data were given as mean \pm SD. Normal distribution was determined using Kolmogorov-Smirnov test. For comparisons between two groups, Student's $t$ test and Mann-Whitney $U$ test were used as appropriate. Spearman correlation test was performed between the variables. $p$ value $<0.05$ was considered statistically significant.

\section{Results}

Table 1 shows the demographic, molecular and clinical features of the patients. We have enrolled 60 Italian genetically proven FD subjects ( 20 men, mean age $46.3 \pm 17.3$ years, mean disease duration 14.9 years). For the sake of completeness, cases numbers 25 and 30, harbouring the D313Y mutation, and cases 40, 41 (Arg 118Cys) remained of no obvious interpretation. In these cases, an extensive work up for other disease explaining organ damage was negative (including NGS screening for genes involved in hypertrophic cardiomyopathy (HCM) in cases 41 ). Family history of cases 40 and 41 was also positive for renal failure and heart diseases in previous generations.

None of the enrolled patients was smoker or heavy drinker, and no additional diseases were reported. In eight cases (numbers 1, 2, 19, 30, 31, 32, 40, 41 Table 1), at the time of the genetic confirmation, multi organ screening 
Table 2 Differences among sex in clinical features of FD

\begin{tabular}{lccl}
\hline & \multicolumn{2}{l}{ Gender } & \\
\cline { 2 - 4 } & $\mathrm{M}$ & $\mathrm{F}$ & $p$ \\
\hline Hypertrophic cardiomyopathy & $14(70 \%)$ & $11(28.2 \%)$ & $\mathbf{0 . 0 0 2}$ \\
Arrhythmia & $8(42.1 \%)$ & $7(17.5 \%)$ & $\mathbf{0 . 0 4 3}$ \\
Myocardial ischemia & $1(5.3 \%)$ & & 0.322 \\
Proteinuria & $14(70 \%)$ & $20(50 \%)$ & 0.141 \\
Renal failure & $2(10 \%)$ & $2(5 \%)$ & 0.595 \\
Stroke & $4(20 \%)$ & $15(37.5 \%)$ & 0.170 \\
Acroparesthesia & $7(35 \%)$ & $17(43.6 \%)$ & 0.525 \\
Vertebrobasilar dolichoectasia & $5(25 \%)$ & $7(17.9 \%)$ & 0.524 \\
Gastrointestinal involvement & $3(15 \%)$ & $10(25.6 \%)$ & 0.351 \\
\hline
\end{tabular}

$M$ male, $F$ female, $P$ p value

Bold values indicate statistically significant

Table 3 Age of onset of different clinical features among FD gender AGE OF ONSET years (standard deviation)

\begin{tabular}{llll}
\hline & Male & Female & $p$ \\
\hline $\begin{array}{l}\text { Hypertrophic cardiomyo- } \\
\text { pathy }\end{array}$ & $44.36(13.62)$ & $52.38(9.46)$ & 0.17 \\
$\begin{array}{l}\text { Arrhythmia } \\
\text { Myocardial ischemia }\end{array}$ & $\begin{array}{l}55.71(13.27) \\
\text { Proteinuria }\end{array}$ & $58.67(14.74)$ & 0.762 \\
$\begin{array}{l}\text { Renal failure } \\
\text { Stroke }\end{array}$ & $41.67(15.33)$ & $39.13(14.81)$ & 0.693 \\
$\begin{array}{l}\text { Acroparesthesia } \\
\text { Vertebrobasilar dolich- } \\
\text { oectasia }\end{array}$ & $43.5(0.71)$ & $37.73(3.49)$ & 0.046 \\
$\begin{array}{l}\text { Gastrointestinal involve- } \\
\text { ment }\end{array}$ & 10 & $26.2(13.79)$ & 0.298 \\
\hline
\end{tabular}

was either negative or revealed a sub-clinical organ impairment.

Hypertrophic cardiomyopathy and arrhythmia were significantly more frequent in male $(p=0.002$ and $p=0.043$ respectively) (Table 2). Age of stroke onset was earlier in females than males ( 37.73 years vs 43.5 years, $p=0.046$ ), whereas gastrointestinal involvement was earlier in males (10 years) than in females (27.43 years, $p=0.02$ ) (Table 3 ).
Table 4 presents values of oxidative stress parameters among patients and controls. As expected, the average values of all oxidative stress parameters in healthy controls were in the normal range, while AOPP levels were significantly higher ( $p<0.00001$, Fig. 1) and FRAP (Fig. 2) and thiols (Fig. 3) significantly lower in FD than healthy controls $(p<0.00001)$. These parameters were not significantly different neither between enzyme replacement therapy (ERT) or non-ERT patients (AOPP: $p=0.129$, FRAP $p=0.441$, thiols $p=0.315$ ) or gender ( $p=0.799$ for AOPP, $p=0.901$ for FRAP, $p=0.367$ for thiols respectively), or age of onset of the disease.

No association was observed between hypertrophic cardiomyopathy, arrhythmia, myocardial ischemia, proteinuria, renal failure, acroparesthesia, vertebrobasilar dolichoectasia, gastrointestinal involvement and oxidative stress parameters. The only observed association was between FRAP levels and stroke $(p=0.02)$, with pathologic levels of FRAP inversely associated with stroke.

No correlation between oxidative stress parameters and overall time of FD was found (AOPP $p=0.324$; FRAP $p=0.192$; thiols $p=0.581$ ). Moreover, no correlation was observed between Lyso-Gb3 levels and AOPP $(p=0.415)$, FRAP $(p=0.51)$ or thiols $(p=0.827)$.

Comparing the classical (onset under 50 years of onset) and late onset FD forms, significant higher values of AOPP levels were observed in the late onset form $(p=0.04$, Table 5). The same was observed in our control group $(<50$ years old vs $>50$ years old, Table 6 ). Comparing late FD forms with the control subgroup over 50 years, a significant difference in the average values of AOPP, FRAP and thiols was found, as shown in Table 7.

Given the difference on age described above, we performed a bivariate analysis, including age, and disease overall time (defined as difference between actual age and age at first disease manifestation) and cardiomyopathy as independent variables; the difference was significant despite correction for age $(p=0.047)$ and disease overall time $(p=0.045)$.

Figure 4 shows oxidative stress parameters over time in a subgroup of eight treatment-naïve patients all with normal Lyso-Gb3 (reference values $<1.8 \mathrm{ng} / \mathrm{mL}$ ) at the enrolment time, at the follow up and the relation with significant clinical or subclinical events. Four patients (4A, B, C and $\mathrm{D})$ showed a significant alteration in oxidative stress
Table 4 Values of oxidative stress (AOPP, FRAP, Thiols) among patients and controls. $\mathrm{RV}$ : reference values. IQR: interquartile range

\begin{tabular}{lccc}
\hline & Median Value (IQR) & Median Value (IQR) & $P$ \\
& Patients & Controls & \\
\hline AOPP (RV 124.5-190.5 nmol/mL) & $342.74(146.15)$ & $185.32(43.93)$ & $<\mathbf{0 . 0 0 0 0 0 1}$ \\
FRAP (RV > 0.7 mmol/L) & $0.61(0.18)$ & $0.78(0.12)$ & $<\mathbf{0 . 0 0 0 0 0 1}$ \\
Thiols (RV 0.4-0.6 mmol/L) & $0.28(0.10)$ & $0.47(0.12)$ & $<\mathbf{0 . 0 0 0 0 0 1}$ \\
\hline
\end{tabular}

Bold values indicate statistically significant 


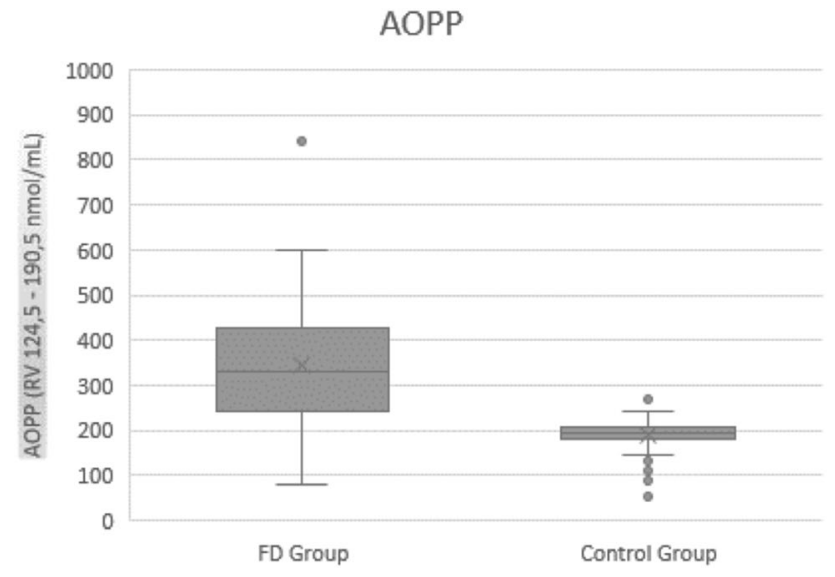

Fig. 1 AOPP values in FD patients and controls

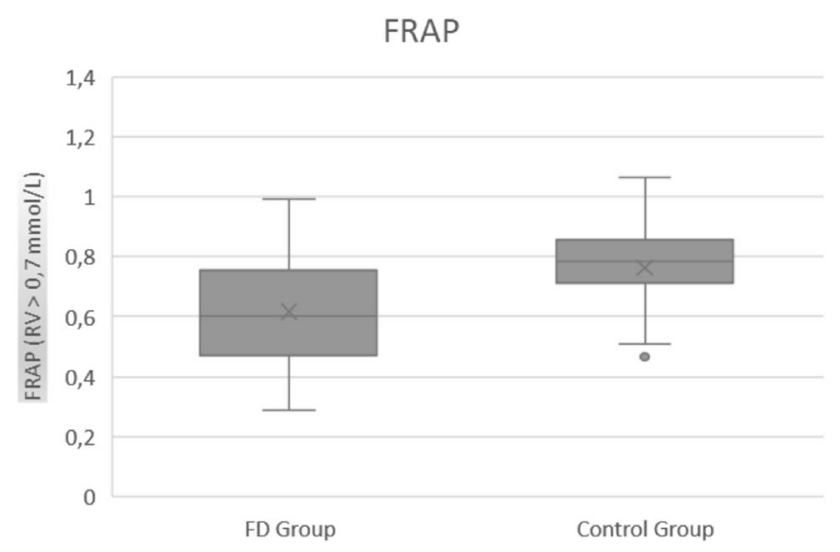

Fig. 2 FRAP values in FD patients and controls

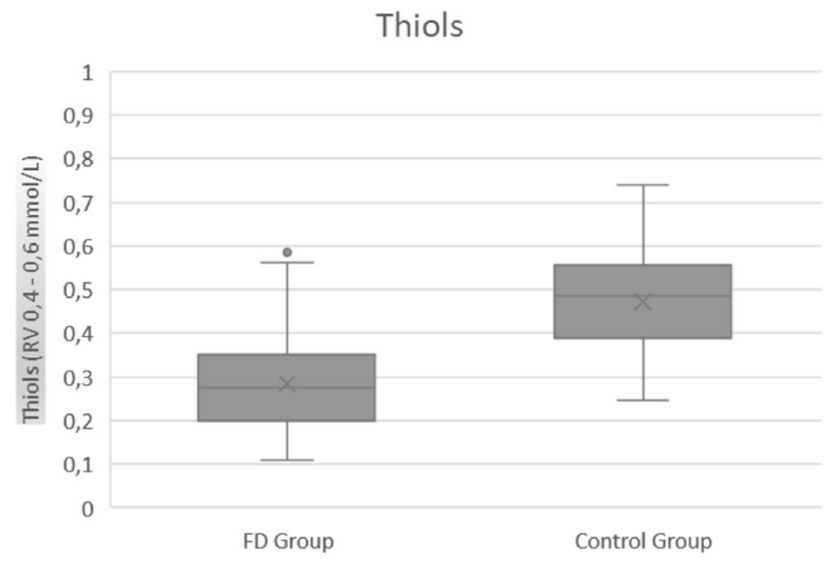

Fig. 3 Thiols values in FD patients and controls
Table 5 Average values of oxidative stress parameters in classic vs late onset FD

\begin{tabular}{lrlll}
\hline & $N$ & Mean & Standard Deviation & $p$ \\
\hline AOPP & & & & \\
Late & 10 & 428.5900 & 187.40270 & $\mathbf{0 . 0 4 0}$ \\
Classic & 48 & 324.8558 & 131.47583 & \\
FRAP & & & & \\
Late & 8 & 0.62338 & 0.139265 & 0.903 \\
Classic & 46 & 0.61476 & 0.189121 & \\
Thiols & & & & \\
Late & 10 & 0.24570 & 0.079635 & 0.227 \\
Classic & 48 & 0.29217 & 0.114189 & \\
\hline
\end{tabular}

Bold value indicates statistically significant

parameters already before the appearance of organ impairment or signs of FD features at the last follow up. Other two patients $(4 \mathrm{E}$ and $4 \mathrm{~F})$ showed pathological oxidative stress parameters already before the appearance of organ damage, while Lyso-Gb3 remained normal. The last two cases (4G and $4 \mathrm{H}$ ) showed pathological oxidative stress parameters and normal Lyso-Gb3 at the time of the diagnosis, having already presented impairment of one or more organ target.

\section{Discussion}

In this study, we observe a significant imbalance of the oxidative stress status in FD patients in different disease stage, including those cases in which Lyso-Gb3 was normal and 1 or organ damage was not yet manifested.

ERT has changed the natural history of the disease in FD patients. Initiation of ERT in childhood could slow, or even stop the progression of organ damage before irreversible changes occur [17]. Although the exact pathogenesis of FD is still under unclear, the accumulation of Gb3 and lysoGb3 in vascular endothelia and subsequent inflammation is thought to play a crucial role, whereas early ERT initiation may reduce lyso-Gb3 deposition and mitigate disease progression [18]. ERT seems to be less effective in patients with both delayed diagnosis and initiation of ERT, and in classic FD there is a tendency to initiate therapy when early symptoms or signs of organ damage occurs [18]. Moreover, in a recent consensus recommendation paper [17] it has been suggested that asymptomatic boys may benefit from an earlier initiation of ERT based on the following criteria: presence of a pathogenic GLA variant responsible for the classic phenotype, family history of disease severity in males, undetectable AGAL-A activity in peripheral blood leukocytes and plasma lyso-Gb3 over $20 \mathrm{nmol} / \mathrm{L}$. Therefore, biomarkers could be used as reliable features to guide the clinician in the therapeutic management. 
Table 6 Average values of oxidative stress parameters in healthy controls $(<50$ ys old vs $>50$ ys old)

\begin{tabular}{lllcccc}
\hline & AGE & $N$ & \multicolumn{1}{l}{ Mean } & Std. Deviation & Std. Error Mean & $p$ \\
\hline AOPP & $\geq 50$ & 58 & 195.7238 & 33.88970 & 4.44994 & $\mathbf{0 . 0 0 7}$ \\
& $<50$ & 19 & 164.9105 & 61.68450 & 14.15139 & \\
FRAP & $\geq 50$ & 58 & 0.78448 & 0.133982 & 0.017593 & $\mathbf{0 . 0 1 4}$ \\
& $<50$ & 19 & 0.69931 & 0.104409 & 0.023953 & \\
Thiols & $\geq 50$ & 58 & 0.48364 & 0.129588 & 0.017016 & 0.154 \\
& $<50$ & 19 & 0.43768 & 0.086380 & 0.019817 & \\
\hline
\end{tabular}

Bold values indicate statistically significant

\begin{tabular}{lccccc}
\hline & $N$ & Mean & Std. Deviation & Std. Error Mean & $p$ \\
\hline AOPP & & & & & \\
$\quad$ Late Form & 10 & 428.5900 & 187.40270 & 59.26194 & $<\mathbf{0 . 0 0 0 0 0 1}$ \\
$\quad$ Controls $\geq 50$ & 58 & 195.7238 & 33.88970 & 4.44994 & \\
FRAP & & & & 0.049238 & $\mathbf{0 . 0 0 2 3 0 9}$ \\
Late Form & 8 & 0.62338 & 0.139265 & 0.017593 & \\
Controls $\geq 50$ & 58 & 0.78448 & 0.133982 & & \\
Thiols & & & & 0.025183 & $\mathbf{0 . 0 0 0 0 0 1}$ \\
Late Form & 10 & 0.24570 & 0.079635 & 0.017016 & \\
Controls $\geq 50$ & 58 & 0.48364 & 0.129588 &
\end{tabular}

Bold values indicate statistically significant
Table 7 Average values of oxidative stress parameters in late onset FD vs healthy controls ( $>50$ years old) p22phox expression and MDA levels), suggesting a possible role of oxidative stress in cardiovascular-renal remodelling pathophysiology [22]. In their work, patients were all in ERT, suggesting that oxidative stress occurs despite ERT in FD related-left ventricular hypertrophy. Similar conclusions were reached by Biancini et al. in 2016 that showed higher lipid peroxidation levels in FD before ERT, which could not be reversed by the treatment [7]. These findings have led to suppose a role for oxidative stress-targeted therapies in addition to ERT in FD.

It has been shown that $\mathrm{Gb} 3$ accumulation is not limited to the lysosomes but also in endothelial cells membranes, inducing deregulating endothelial NO synthase activity with reduced NO production that favours oxidative stress [23].

Our results confirm oxidative stress activation in FD; in particular, we have observed high levels of AOPP and reduced levels of antioxidants (FRAP and thiols) in patients compared to healthy controls; these parameters were altered despite ERT, confirming Biancini observation [7]. Given the others study on oxidative stress published up so far, we might assume a vicious cycle between Lyso-Gb3 deposition and oxidative stress: Lyso-Gb3 deposition induces organ damage, endothelial dysfunction, increases vascular superoxide and free radical formation, oxidative DNA damage and altered oxidative response, which in turn enhances organ damage and remodelling.

Recently, Ravarotto et al. have documented oxidative stress activation and oxidative stress signalling (higher 
Fig. 4 Oxidative stress parameters (blue: Thiols, orange: FRAP, red: AOPP) and LysoGb3 (yellow) values over time. The green area and dashed lines show reference values (RV) $(\mathrm{AOPP}<190.5 \mathrm{nmol} /$ $\mathrm{mL} ;$ Lyso-Gb3 $<1.8 \mathrm{ng} /$ $\mathrm{mL}$; Thiols $>0,4 \mathrm{mmol} / \mathrm{L}$; FRAP $>0.7 \mathrm{mmol} / \mathrm{L})$. RV are obtained from a population of 77 healthy controls (32 men, mean age $61.5 \pm 18.2$ years), unrelated to the patients, recruited at the coordinator centre. The vertical dashed line indicates the appearance of a clinical event related to FD natural history

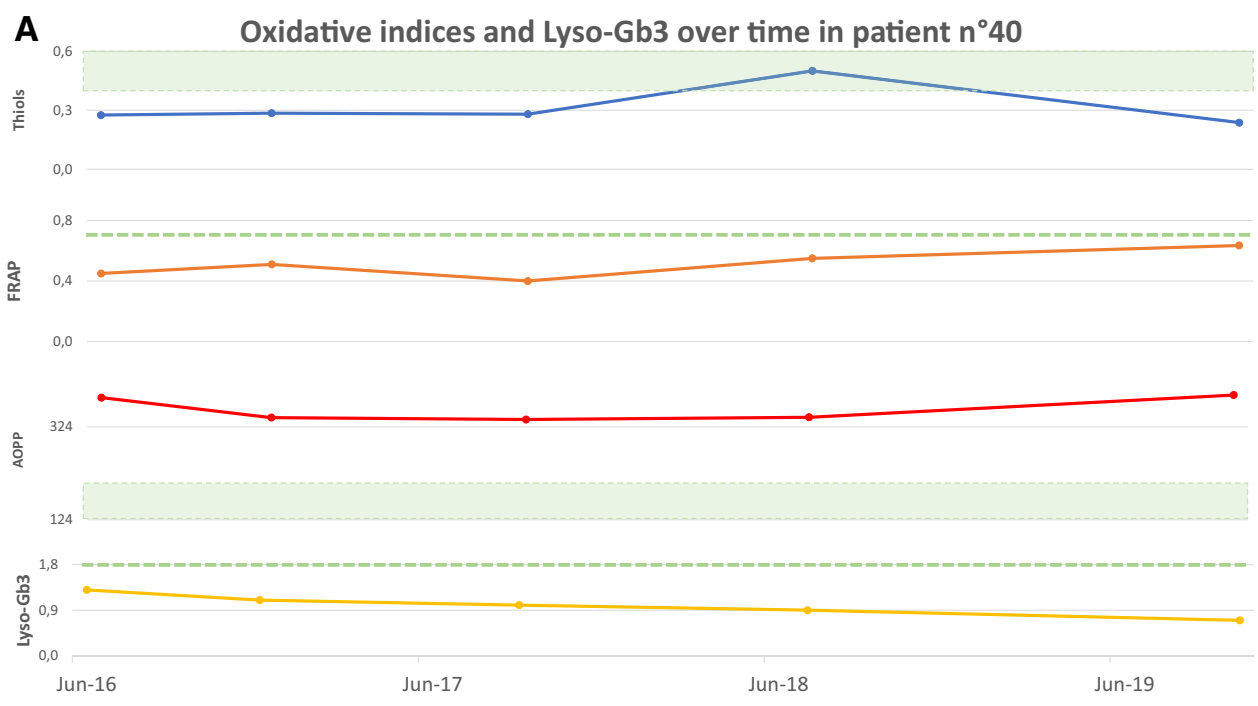

B Oxidative indices and Lyso-Gb3 over time in patient $\mathrm{n}^{\circ} 1$

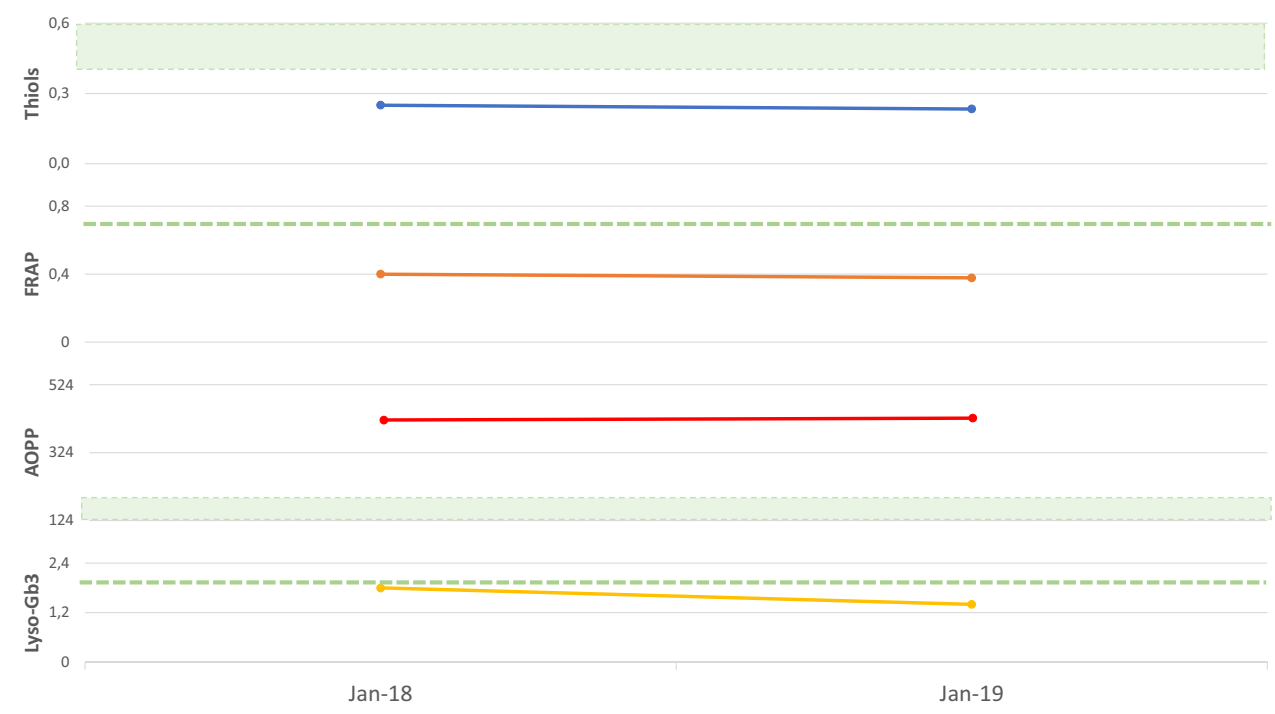

We have not observed an association between oxidative stress parameters alteration and disease manifestations. The inverse association between pathological values of FRAP and stroke (the opposite of what one would expect, given the possible role of oxidative stress in cerebral small vessels disease) is unclear and requires further investigation.

We also found significantly higher levels of AOPP between late onset and classic FD, despite correction for age and disease overall time.

Interestingly, in a group of eight treatment-naïve subjects/ patients with FD-related mutations and normal Lyso-Gb3 levels we have found pathological oxidative stress parameters. Lyso-Gb3 levels didn't correlate with the evaluated biomarkers; we can assume that these parameters reflect early activation of oxidative stress and organ damage, before lysoGb3 elevation, but further studies are needed. This group consisted of some challenging cases: two pre-symptomatic young females with the pathogenic p.Phe113Leu, traditionally related to a late-onset phenotype, in which lysoGB3 levels could be sometimes normal [24]; a 10-year-old male with a classical phenotype and normal levels of LysoGB3; two females with the recently considered "likely benign" 
Fig. 4 (continued)
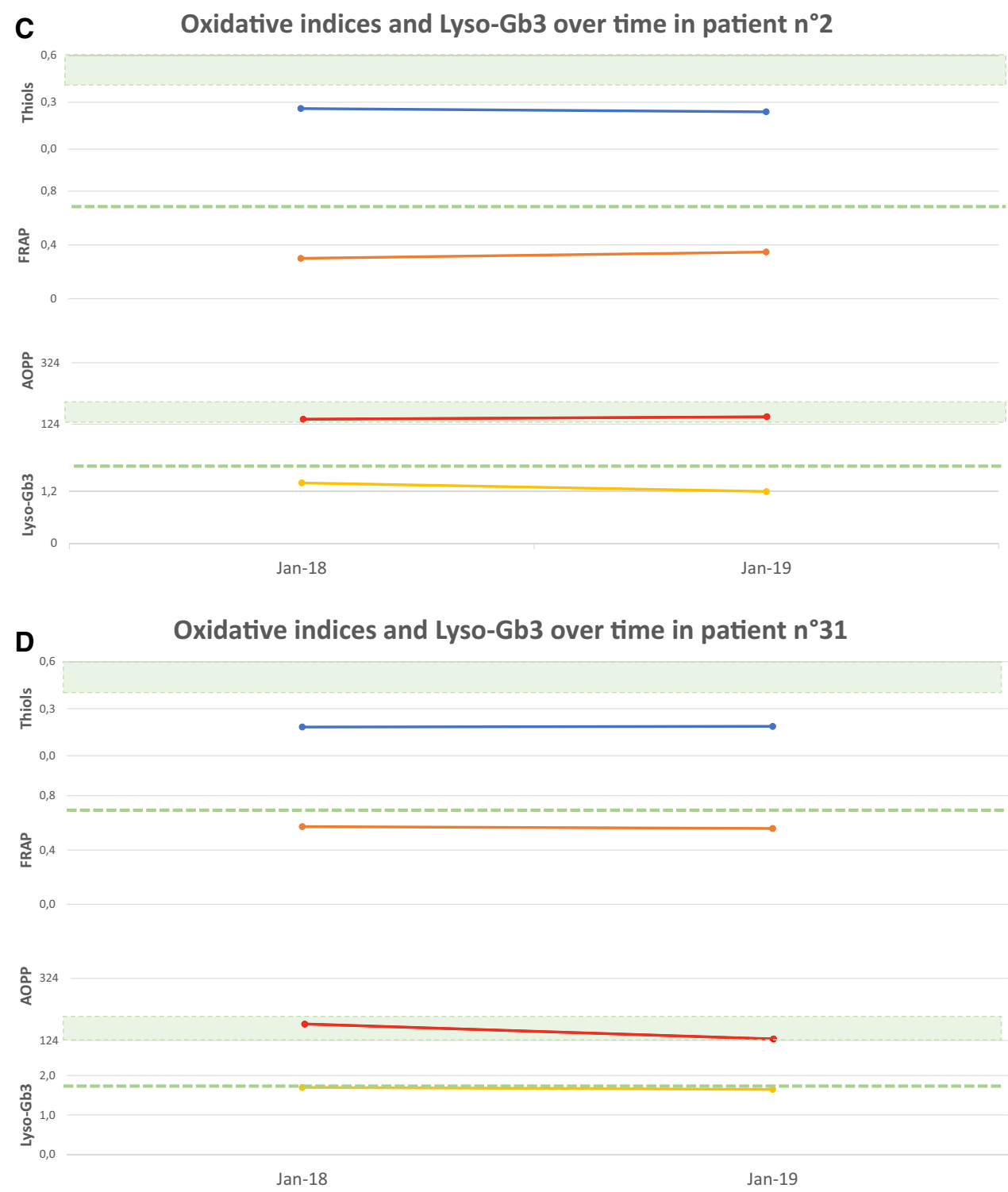

variant D313Y but manifesting some clinical manifestations of FD [25, 26]; a 18-year-old male with HCM, and his asymptomatic mother, with the Arg118Cys variant with conflicting interpretations of pathogenicity according to ClinVar [27-29]. These cases rise some queries about the diagnostic value of lyso-GB3 in some settings, and confirms that diagnosis of FD cannot be made solely on genetic basis. Histopathologic studies are crucial in challenging cases to confirm the diagnosis of FD but may be invasive (as in myocardial biopsy), and not always available in some settings. Unfortunately, we do not have histopathological studies in our cases. The altered parameters of oxidative stress in the above cases may suggest an early involvement of redox imbalance in FD-induced organ damage, disease onset and progression. Although some authors have demonstrated the progression of organ damage in ERT patients, the early treatment is considered to be the key to dramatically change the natural history of disease in these patients [18, 30, 31]. Our results are not enough to state that ERT should be started in subjects with FD-related mutations who do not have signs or symptoms of disease but signs of oxidative stress, however they pave the way for further studies. To date, beside AGALA activity in males, the only surrogate biomarker recognized in FD is Lyso-Gb3. Considering what we have observed, specific oxidative stress markers could be even more sensitive than Lyso-Gb3 in the early phase of the disease, thus 
Fig. 4 (continued)
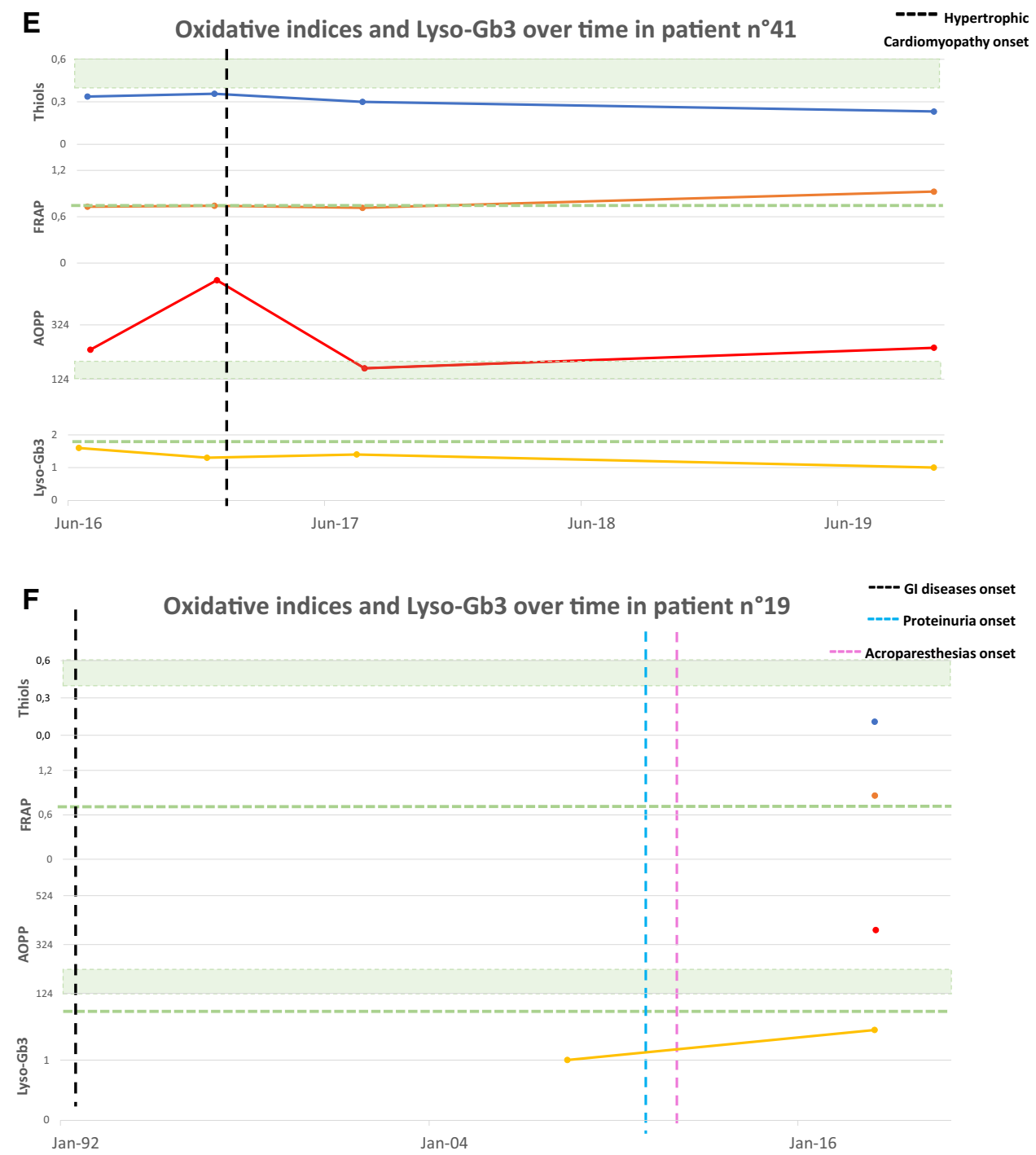

representing new early biomarkers with a potential role in helping diagnosis and time to treatment decision.

Our study has some limitations. We have enrolled patients at different stage of disease, and the timing of the biomarkers analysis was variable from patient to patient. Moreover, most of our cases are females, thus we could not correlate AGALA activity with the other findings. No complete data about all possible comorbidities were available, although patients didn't have significant comorbidities such as cancer or other inflammatory disorders.
In conclusion, oxidative stress occurs in FD in both treated and naive patients, highlighting the need of further collaborative research in oxidative stress-targeted therapies in addition to ERT in order to further slow disease progression. Furthermore, oxidative stress biomarkers may represent early markers of disease in treatment-naïve patients with a potential role in helping interpretation of FD-related mutations and time to treatment decision. 
Fig. 4 (continued)
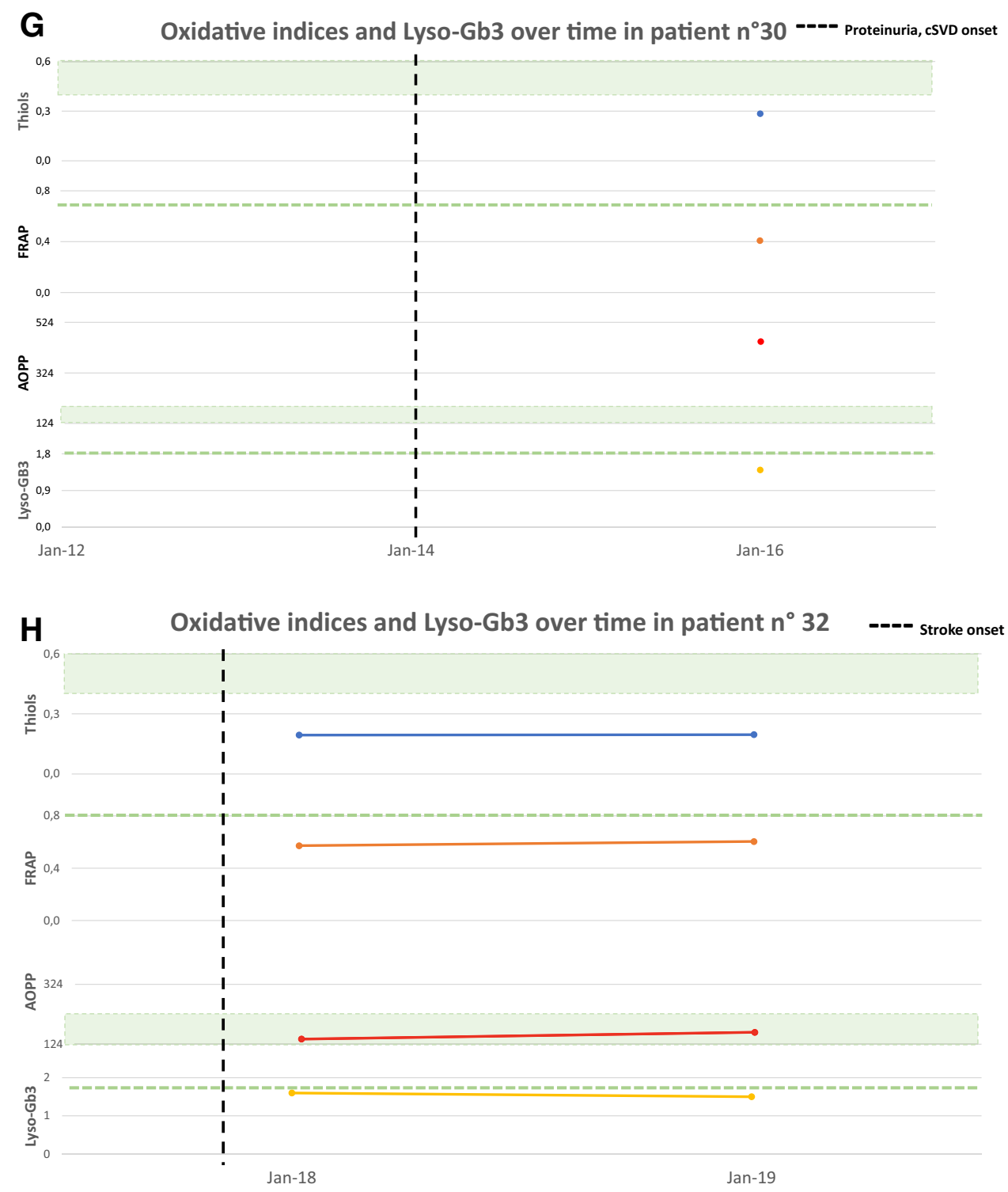

Acknowledgments Open access funding provided by University of Pisa within the CRUI-CARE Agreement. MM was partially supported by an Investigator-Initiated Research grant (IIR-ITA-001466) from Shire International $\mathrm{GmbH}$, a Takeda company.

Author contributions All the authors made substantive intellectual contributions to the database development, data collection, and interpretation.

\section{Compliance with ethical standards}

Conflicts of interest The authors declare that there is no conflict of interest regarding the publication of this paper

Ethical approval The authors report no disclosures relevant to the manuscript.
Open Access This article is licensed under a Creative Commons Attribution 4.0 International License, which permits use, sharing, adaptation, distribution and reproduction in any medium or format, as long as you give appropriate credit to the original author(s) and the source, provide a link to the Creative Commons licence, and indicate if changes were made. The images or other third party material in this article are included in the article's Creative Commons licence, unless indicated otherwise in a credit line to the material. If material is not included in the article's Creative Commons licence and your intended use is not permitted by statutory regulation or exceeds the permitted use, you will need to obtain permission directly from the copyright holder. To view a copy of this licence, visit http://creativecommons.org/licenses/by/4.0/.

\section{References}

1. Germain DP (2010) Fabry disease. Orph J Rare Dis 5:30 
2. Arends M, Wanner C, Hughes D, Mehta A, Oder D, Watkinson OT et al (2017) Characterization of classical and nonclassical fabry disease: A multicenter study. J Am Soc Nephrol 28(5):1631-1641

3. Moore DF, Scott LTC, Gladwin MT, Altarescu G, Kaneski C, Suzuki K et al (2011) Regional cerebral hyperperfusion and nitric oxide pathway dysregulation in fabry disease: reversal by enzyme replacement therapy. Circulation 104(13):1506-1512

4. Moore DF, Ye F, Brennan M-L, Gupta S, Barshop BA, Steiner RD, et al (2004) Ascorbate decreases Fabry cerebral hyperperfusion suggesting a reactive oxygen species abnormality: an arterial spin tagging study. J Magn Reson Imaging 20(4):674-683

5. Shen J-S, Meng X-L, Moore DF, Quirk JM, Shayman JA, Schiffmann R et al (2008) Globotriaosylceramide induces oxidative stress and up-regulates cell adhesion molecule expression in Fabry disease endothelial cells. Mol Genet Metab 95(3):163-168

6. Müller KB, Galdieri LC, Pereira VG, Martins AM, D’Almeida V (2012) Evaluation of oxidative stress markers and cardiovascular risk factors in Fabry Disease patients. Genet Mol Biol 35(2):418-423

7. Biancini GB, Jacques CE, Hammerschmidt T, de Souza HM, Donida B, Deon M et al (2016) Biomolecules damage and redox status abnormalities in Fabry patients before and during enzyme replacement therapy. Clin Chim Acta 461:41-46

8. Chen K-H, Chou Y-C, Hsiao C-Y, Chien Y, Wang K-L, Lai Y-H et al (2017) Amelioration of serum 8-OHdG level by enzyme replacement therapy in patients with Fabry cardiomyopathy. Biochem Biophys Res Commun 486(2):293-299

9. Biancini GB, Morás AM, Reinhardt LS, Busatto FF, de Moura Sperotto ND, Saffi J et al (2017) Globotriaosylsphingosine induces oxidative DNA damage in cultured kidney cells. Nephrology (Carlton) 22(6):490-493

10. Ravarotto V, Simioni F, Carraro G, Bertoldi G, Pagnin E, Calò L (2018) Oxidative stress and cardiovascular-renal damage in fabry disease: is there room for a pathophysiological involvement? J Clin Med 7(11):409

11. Witko-Sarsat V, Friedlander M, Nguyen Khoa T, CapeillèreBlandin C, Nguyen AT, Canteloup S et al (1998) Advanced oxidation protein products as novel mediators of inflammation and monocyte activation in chronic renal failure. J Immunol 161(5):2524-2532

12. Chen YH, Shi W, Liang XL, Liang YZ, Fu X (2011) Effect of blood sample type on the measurement of advanced oxidation protein products as a biomarker of inflammation and oxidative stress in hemodialysis patients. Biomarkers 16(2):129-135

13. Selmeci L (2011) Advanced oxidation protein products (AOPP): novel uremic toxins, or components of the non-enzymatic antioxidant system of the plasma proteome? Free Radic Res 45:1115-1123

14. Benzie IFF, Strain JJ (1996) The ferric reducing ability of plasma (FRAP) as a measure of « antioxidant power » : The FRAP assay. Anal Biochem 239(1):70-76

15. Nowak M, Świętochowska E, Wielkoszyński T, Marek B, Karpe J, Górski J et al (2003) Changes in blood antioxidants and several lipid peroxidation products in women with age-related macular degeneration. Eur J Ophthalmol 13(3):281-286

16. Hu ML (1994) Measurement of protein thiol groups and glutathione in plasma. Methods Enzymol 233(C):380-385

17. Germain DP, Fouilhoux A, Decramer S, Tardieu M, Pillet P, Fila $\mathrm{M}$ et al (2019) Consensus recommendations for diagnosis, management and treatment of Fabry disease in paediatric patients. Clin Genet 96(2):107-117
18. Kritzer A, Siddharth A, Leestma K, Bodamer O (2019) Early initiation of enzyme replacement therapy in classical Fabry disease normalizes biomarkers in clinically asymptomatic pediatric patients. Mol Genet Metab reports 21:100530

19. Biancini GB, Vanzin CS, Rodrigues DB, Deon M, Ribas GS, Barschak AG et al (2012) Globotriaosylceramide is correlated with oxidative stress and inflammation in Fabry patients treated with enzyme replacement therapy. Biochim Biophys Acta 1822(2):226-232

20. Simoncini C, Chico L, Concolino D, Sestito S, Fancellu L, Boadu W et al (2016) Mitochondrial DNA haplogroups may influence Fabry disease phenotype. Neurosci Lett. 629:58-61

21. Chimenti C, Scopelliti F, Vulpis E, Tafani M, Villanova L, Verardo R, et al (2015) Increased oxidative stress contributes to cardiomyocyte dysfunction and death in patients with Fabry disease cardiomyopathy. Hum Pathol 46(11):1760-1768

22. Ravarotto V, Carraro G, Pagnin E, Bertoldi G, Simioni F, Maiolino $\mathrm{G}$ et al (2018) Oxidative stress and the altered reaction to it in Fabry disease: A possible target for cardiovascular-renal remodeling? PLoS One 13(9):e0204618

23. Shu L, Vivekanandan-Giri A, Pennathur S, Smid BE, Aerts JMFG, Hollak CEM et al (2014) Establishing 3-nitrotyrosine as a biomarker for the vasculopathy of Fabry disease. Kidney Int 86(1):58-66

24. Smid BE, Van der Tol L, Biegstraaten M, Linthorst GE, Hollak CEM, Poorthuis BJHM (2015) Plasma globotriaosylsphingosine in relation to phenotypes of fabry disease. J Med Genet 52(4):262-268

25. Yasuda M, Shabbeer J, Benson SD, Maire I, Burnett RM, Desnick RJ (2003) Fabry disease: characterization of $\alpha$-galactoslidase a double mutations and the D313Y Plasma enzyme pseudodeficiency allele. Hum Mutat 22(6):486-492

26. Froissart R, Guffon N, Vanier MT, Desnick RJ, Maire I (2003) Fabry disease: D313Y is an alpha-galactosidase A sequence variant that causes pseudodeficient activity in plasma. Mol Genet Metab 80(3):307-314

27. Golbus JR, Puckelwartz MJ, Dellefave-Castillo L, Fahrenbach JP, Nelakuditi V, Pesce LL et al (2014) Targeted analysis of whole genome sequence data to diagnose genetic cardiomyopathy. Circ Cardiovasc Genet 7(6):751-759

28. Ferreira S, Ortiz A, Germain DP, Viana-Baptista M, CaldeiraGomes A, Camprecios M et al (2015) The alpha-galactosidase A p.Arg 118Cys variant does not cause a Fabry disease phenotype: data from individual patients and family studies. Mol Genet Metab 114(2):248-258

29. Turaça LT, Pessoa JG, Motta FL, Muñoz Rojas MV, Müller KB, Lourenço CM et al (2012) New mutations in the GLA gene in Brazilian families with Fabry disease. J Hum Genet 57:347-351

30. Germain DP, Arad M, Burlina A, Elliott PM, Falissard B, FeldtRasmussen U et al (2019) The effect of enzyme replacement therapy on clinical outcomes in female patients with Fabry disease-a systematic literature review by a European panel of experts. Mol Genet Metab 126(3):224-235

31. Germain DP, Elliott PM, Falissard B, Fomin VV, Hilz MJ, Jovanovic A et al (2019) The effect of enzyme replacement therapy on clinical outcomes in male patients with Fabry disease: a systematic literature review by a European panel of experts. Mol Genet Metab Rep 19:100454 